Archives

Dénombrements officiers « moyens » (III)/Varia

\title{
Officiers « moyens »
}

Monarchie administrative et villes à l'aune du dénombrement des officiers royaux en situation de cumul au XVIII ${ }^{\mathrm{e}}$ siècle

\section{Vincent Meyzie}

\section{(2) OpenEdition}

\section{Journals}

Édition électronique

URL : http://journals.openedition.org/ccrh/3276

DOI : $10.4000 /$ ccrh.3276

ISSN : 1760-7906

Éditeur

Centre de recherches historiques - EHESS

Édition imprimée

Date de publication : 1 juillet 2006

Pagination : $99-125$

ISSN : 0990-9141

Référence électronique

Vincent Meyzie, "Officiers « moyens » », Les Cahiers du Centre de Recherches Historiques [En ligne],

38 | 2006, mis en ligne le 28 septembre 2011, consulté le 01 mai 2019. URL : http://

journals.openedition.org/ccrh/3276 ; DOI : 10.4000/ccrh.3276

Ce document a été généré automatiquement le 1 mai 2019.

Article L.111-1 du Code de la propriété intellectuelle. 


\title{
Officiers « moyens »
}

\author{
Monarchie administrative et villes à l'aune du dénombrement des \\ officiers royaux en situation de cumul au XVIII ${ }^{\mathrm{e}}$ siècle
}

\section{Vincent Meyzie}

1 Les dénombrements des officiers royaux dans la France moderne constituent des préoccupations classiques pour les grands serviteurs de la monarchie et les historiens. Liés à des motivations pratiques chez les premiers, ils visent à mieux connaître la répartition des administrateurs disponibles pour le gouvernement du royaume, voire à programmer une réduction éventuelle de leur effectif à l'instar de Colbert jugeant pléthorique dans la décennie 1660 le nombre d'officiers royaux de justice ${ }^{1}$. Fondés sur des interrogations heuristiques chez les seconds, ils ont pour but d'évaluer le poids de l'État d'offices au sein de l'État royal ou d'estimer la place des officiers royaux parmi les divers groupes composant la société urbaine ${ }^{2}$. Marquées par une forte dimension comptable, ces tentatives de pesées globales à l'échelle étatique ou citadine reposent largement sur la documentation générée par l'État, avec notamment le recours aux dénombrements d'ensemble telle l'enquête de 1515 ou l'enquête de 1665 initiée par Colbert ${ }^{3}$. Toutefois, la mobilisation surtout quantitative de sources administratives à caractère massif mais aussi univoque élude en partie les difficultés inhérentes aux mécanismes subtils de la vénalité des offices d'une part et à la diversité des pratiques officières d'autre part. En premier lieu, elle délaisse la différence entre le nombre des offices et l'effectif des officiers, liée à la fois au statut des charges - avec des cas fréquents de réunion plus ou moins durable au corps ou sur la tête d'un même individu - et à leur situation conjoncturelle, avec la distinction entre offices pourvus et vacants. En second lieu, elle néglige l'influence déterminante des comportements officiers marqués par les phénomènes usuels d'officiers adeptes d'un absentéisme chronique ou en situation de cumul des fonctions publiques.

2 Au début du XVIII siècle, le lieutenant général du présidial de Rouen Pierre Le Pesant de Boisguilbert - davantage connu pour ses conceptions économiques préfigurant le libéralisme économique ${ }^{4}$ - apparait ainsi comme un officier cumulant remarquable au sein du tribunal normand. Outre sa charge de chef de la sénéchaussée acquise en 1690, il possède la lieutenance générale de police depuis 1699 et détient aussi les deux charges de 
président du siège grâce à l'acquisition en $1705 \mathrm{du}$ second office de président ${ }^{5}$. Représente-t-il le type de l'officier «moyen» cumulant comme détenteur de quatre fonctions majeures dans la cité ? En raison du nombre des offices possédés et du montant élevé de sa taxation le classant au sommet de la hiérarchie des imposés, son cas apparaît plus atypique qu'exemplaire. En revanche, son absence du paiement de la somme exigée s'inscrit dans un comportement majoritaire, à l'instar de l'important effectif des cent quarante-cinq individus recensés ${ }^{6}$. Si la comparaison de sa situation à celle des autres officiers de Normandie demeure difficilement envisageable du fait de l'imprécision de la liste établie, l'exemple de Boisguilbert incite toutefois à évaluer l'extension des pratiques cumulatives dans d'autres provinces.

3 En effet, dans le cas des officiers «moyens", un ensemble documentaire concernant le début du XVIII siècle permet de prendre en compte à la fois la préoccupation légitime d'une évaluation comptable, les écueils méthodologiques liés au système de la « vénalité légale $»^{7}$ et la question majeure des postures officières. Il porte sur les officiers royaux des généralités de Limoges, Riom et La Rochelle en situation de cumul, soumis à un recensement en vertu d'un édit de mars 1709. Les listes conservées offrent l'opportunité d'analyser la place des officiers «moyens " parmi les administrations et les villes des trois provinces. Appréhendées dans la perspective d'une histoire sociale des institutions ${ }^{8}$, elles dévoilent non seulement un état partiel de la démographie des offices et des officiers à l'orée du siècle mais aussi les positions adoptées par les administrateurs et les serviteurs de la monarchie vis-à-vis du dénombrement et du cumul des charges royales. Ce dossier présente un double avantage par sa chronologie. Tout d'abord, il s'inscrit en amont de la période 1715-1740, mieux connue grâce aux enquêtes initiées par le contrôleur général Orry et le chancelier d'Aguesseau'. Il témoigne ensuite d'un moment fondamental dans l'histoire des offices «moyens »- une phase d'expansion maximale à la fin du règne de Louis XIV - avec la mise en place en 1709 d'une politique fiscale de taxation des officiers royaux en situation de cumul.

Un double questionnement permet d'analyser ce dossier comme révélateur des comptabilités officielles mais aussi des pratiques étatiques et officières. Les officiers «moyens » occupent-ils une position spécifique parmi les officiers royaux en situation de cumul au début du XVIII siècle? L'approche quantifiée demeure nécessaire dans un premier temps afin de déterminer leur éventuelle place privilégiée au sein des administrateurs de rang intermédiaire et de l'estimer à l'aune de l'équipement institutionnel des villes. Dans un second temps, une lecture plus qualitative interprète le phénomène du cumul comme élément significatif des relations entre l'État royal et ses serviteurs, en particulier dans le cadre du renforcement de sa politique vénale et l'élargit ensuite à ses significations professionnelles et sociales en le replaçant dans les trajectoires officières. Quelles positions normatives, pratiques et financières adoptent la monarchie administrative et ses représentants vis-à-vis du cumul ? Quelles motivations précises fondent les pratiques cumulatives des officiers « moyens»?

\section{La place des officiers « moyens » parmi les officiers royaux en situation de cumul}

5 L'établissement de nomenclatures nominatives à caractère fiscal à la fin du règne de Louis XIVrend possible une appréhension globale du cumul des offices royaux pour les trois 
généralités de Riom, Limoges et La Rochelle ${ }^{10}$. Présentées sous la forme d'une liste au brouillon pour la province auvergnate ou sous l'aspect de tableaux définitifs dans les deux autres cas, elles permettent d'identifier les officiers cumulants et les types d'offices détenus. Anciennes et de taille similaire, les généralités de Limoges et de Riom correspondent à des provinces enclavées et à l'urbanisation lâche. Créée en 1694 par la réunion de l'Aunis et de la Saintonge, la généralité récente de La Rochelle présente un profil différent, marqué par la fonction commerciale et la vocation maritime avec notamment le port rochelais mais aussi par la spécificité militaire avec des places fortes comme Brouage. Plusieurs offices témoignent, sur la liste de 1712, de la place tenue par l'administration de la marine ${ }^{11}$. Une seconde raison, d'ordre historiographique, conduit par ailleurs à privilégier les cas des généralités de Limoges et de Riom, mieux desservies par plusieurs études spécifiques sur les serviteurs du roi de rang intermédiaire ${ }^{12}$.

6 Le tableau 1 présente une approche synchronique du nombre des officiers royaux cumulants et de leur répartition institutionnelle à la fin du règne de Louis XIV dans les trois généralités.

Tableau 1. Les officiers cumulants dans les généralités de Limoges, Riom et La Rochelle (1709-1712)

\begin{tabular}{|c|c|c|c|c|c|c|c|c|c|c|}
\hline & institutio & ns avec des offic & iers « moyens" & & & & & & & \\
\hline $\begin{array}{l}\text { répartition } \\
\text { par } \\
\text { généralité }\end{array}$ & Présidial & $\begin{array}{l}\text { Sénéchaussée, } \\
\text { baillage }\end{array}$ & maréchaussée & élection & $\begin{array}{l}\text { Maîtrise } \\
\text { des } \\
\text { eaux et } \\
\text { forêts, } \\
\text { greniers } \\
\text { à sel }\end{array}$ & $\begin{array}{l}\text { Total des } \\
\text { officiers } \\
\text { «moyens }\end{array}$ & $\begin{array}{l}\text { Bureau } \\
\text { des } \\
\text { finances }\end{array}$ & Prévôté & divers & total \\
\hline Limoges & 17 & 1 & 2 & 6 & / & 26 & 4 & 4 & 6 & 40 \\
\hline Riom & 12 & 4 & 1 & 5 & 2 & 24 & 1 & 3 & $15(a)$ & 43 \\
\hline $\begin{array}{l}\text { La } \\
\text { Rochelle }\end{array}$ & 6 & / & 1 & 9 & 1 & 17 & 0 & 8 & $29(\mathrm{~b})$ & 54 \\
\hline Total & 35 & 5 & 4 & 20 & 3 & 67 & 5 & 15 & 50 & 137 \\
\hline
\end{tabular}

a) : ce nombre inclut 3 conseillers de la Cour des aides de Riom.

b) : ce nombre élevé d'officiers « divers » en situation de cumul dans la généralité de La Rochelle résulte notamment des effectifs significatifs d'officiers subalternes comme les procureurs et les greffiers (avec 8 cas) et des offices de receveurs (avec 6 cas).

7 Focalisé sur la catégorie des officiers « moyens » comprise dans son acceptation stricte, il exclut les officiers des bureaux des finances et des justices royales ordinaires ${ }^{13}$.

Ce tableau montre l'importance numérique des officiers «moyens » cumulants dans les trois généralités, avec presque la moitié des cas recensés. Une distinction majeure sépare d'une part le Limousin et l'Auvergne, deux provinces dans lesquelles ils représentent une nette majorité des officiers en situation de cumul et d'autre part l'Aunis et la Saintonge où ils constituent seulement une forte minorité. Le moindre équipement institutionnel de la généralité de La Rochelle, privée de bailliage et comportant seulement les deux 
présidiaux de La Rochelle et de Saintes explique en grande partie la différence. Le tableau 1 dévoile une seconde caractéristique majeure de leur profil : la surreprésentation notable de la magistrature, en particulier des juges présidiaux. Ils représentent à eux seuls la moitié ou plus du groupe dans les généralités de Limoges et de Riom: six magistrats sont concernés dans le siège de Tulle, cinq à Angoulême, quatre à Limoges et trois à Brive tandis que six juges se trouvent en situation de cumul dans le tribunal de Riom, quatre à Clermont et deux à Aurillac (tableaux I et II).

Peut-on distinguer des pratiques cumulatives récurrentes, des types dominants d'association de charges chez les officiers «moyens»? Cette approche nécessite de traiter de manière séparée officiers de finance et de judicature.

Les officiers des élections obéissent à une logique majoritaire et simple de cumul définie par la spécialisation financière. En effet, sur vingt cas relevés, huit d'entre eux possèdent les charges anciennes et alternatives de receveurs des tailles et dix autres cumulent une recette des tailles avec des offices annexes de finance ou de receveurs subalternes. À Angoulême, Taboureau détient ainsi les deux charges de receveurs des tailles tandis qu'à Cognac, Jérémie Simon associe la recette des tailles à celle des deniers communs et octrois. Deux cas seulement apparaissent atypiques par l'association d'un office «moyen » de finance à une charge subalterne fort différente : le président de l'élection Nicolas Dufaur et le lieutenant criminel et vérificateur des rôles Jacques Dufaur à Marennes sont aussi notaires royaux.

Deux remarques complémentaires précisent la description: les officiers des élections privilégient le cumul interne à l'institution comme Grenier élu et vérificateur des rôles à Mauriac ; la possession concomitante de deux offices de receveurs des tailles constitue en fait un cumul plus apparent que véritable en raison de leur nature similaire ${ }^{14}$. Cette logique privilégiée de spécialisation financière traduit la préférence accordée à des offices rentables comme capital et source de revenus. Les charges de receveurs des tailles souvent cumulées sont logiquement les plus appréciées pour leur valeur : elles sont ainsi estimées en 1665 à Limoges et à Tulle respectivement à 47000 livres et 40000 livres $^{15}$.

Les officiers "moyens» de justice s'inscrivent en revanche dans une logique de diversification officière par la grande variété des cumuls pratiqués. Leur présence concomitante dans de nombreuses institutions démontre leur ubiquité remarquable et originale (tableaux I, II et III). Dans la généralité de Limoges, les magistrats présidiaux sont implantés dans sept institutions : la maréchaussée, l'hôtel de la monnaie, le bureau des finances, la police urbaine, le corps de ville grâce aux offices vénaux municipaux, l'élection et la monarchie administrative par les charges vénales de subdélégués. Dans la généralité de Riom, ils sont aussi présents dans sept institutions. Comme dans le Limousin, les juges auvergnats détiennent une position de cumul dans les cinq premières administrations citées; en revanche, ils sont absents des élections et des subdélégations mais présents dans la maîtrise des eaux et forêts de Riom et la Cour des aides de Clermont. Dans la généralité de La Rochelle, ils occupent en parallèle une charge dans quatre institutions : la maréchaussée, la police urbaine, le corps de ville et la monarchie administrative. Dans les provinces d'Auvergne, d'Aunis et de Saintonge, l'étanchéité professionnelle entre les présidiaux et les élections apparaît avec netteté. Elle dénote, notamment dans le cas de la généralité de La Rochelle, une séparation forte entre le monde robin et le milieu financier, ce dernier constituant sans doute une filière de promotion privilégiée pour des individus issus de la marchandise ${ }^{16}$. 

de justice cumulants délimitent le noyau dur d'une élite administrative provinciale. Identifiée par le critère du cumul des fonctions publiques, elle repose sur l'insertion simultanée et diversifiée dans l'appareil étatique. Ces pratiques cumulatives attestent en fait de la permanence de formes traditionnelles d'intégration à l'État d'offices perçu comme source privilégiée d'autorité, de rentabilité et d'honorabilité et de la persistance de serviteurs polyvalents du roi. Elles marquent ainsi une limite fondamentale aux lectures exclusivement néo-webériennes de l'État dans la France du XVIII siècle insistant sur sa neutralité et sa spécialisation ${ }^{17}$. En effet, elles s'inscrivent en décalage à la fois par rapport au développement d'une élite administrative désintéressée et par rapport à l'expansion des administrations spécialisées. Les travaux de Marie-Laure Legay sur des administrateurs du Nord de la France illustrent la première approche par la mise en exergue d'un engagement pour la res publica multiple dans ses modalités mais sincère dans ses motivations, engagement contribuant à la formation d'une culture administrative commune ${ }^{18}$. L'analyse du corps des inspecteurs des manufactures par Philippe Minard incarne parfaitement la seconde lecture. Elle montre pour ce personnel technique la mise en place progressive d'une réglementation stricte des carrières avec notamment l'interdiction, contrôlée de manière efficace, d'exercer simultanément d'autres activités ${ }^{19}$. Reposant sur une conception exclusive du service royal, cette spécialisation professionnelle croissante forme une composante essentielle de la modernisation étatique, propre au siècle des Lumières. En regard du modèle émergent du "pré-fonctionnaire» désintéressé et spécialisé, l'ubiquité originale des officiers «moyens » de judicature indique non seulement une évolution décalée au sein de l'État du XVIII siècle mais aussi la conservation parallèle de la conception classique d'une élite administrative polymorphe.

Plusieurs types de cumul apparaissent récurrents chez la magistrature seconde: l'implantation concomitante dans la maréchaussée, la police urbaine, les corps de ville et les subdélégations. Dans le premier cas, elle reflète en partie la situation de la juridiction de maintien de l'ordre en milieu rural peu de temps avant sa réforme en 1720 qui instaure une nouvelle répartition territoriale fondée sur la hiérarchie urbaine et un maillage plus régulier ${ }^{20}$. Les trois autres modalités de carrières simultanées participent d'une évolution commune: l'expansion de la "vénalité légale» dans la seconde moitié du règne de Louis XIV. Elle se traduit en particulier par la création d'offices municipaux vénaux à partir de 1692, la mise en place en 1699 des lieutenances générales de police dans les cités de province et l'érection en 1704 en titres d'offices des subdélégués ${ }^{21}$. Dans quelle mesure les officiers «moyens » de judicature participent-ils à ces nouvelles formes de vénalité, corrélatives du renforcement de la monarchie administrative?

Leur intégration à la maréchaussée prend la forme d'offices couplés et correspond à un cumul imposé. Reposant sur la délivrance d'une lettre de provision unique, elle associe dans cinq villes (Tulle, La Rochelle, Riom, Clermont et Aurillac) les charges de procureur du roi au présidial et à la maréchaussée mais aussi, dans les deux premières cités, les offices de conseiller et d'assesseur. Dans la capitale du Bas-Limousin, Jean-Martial de Fénis de Lacombe détient ainsi l'office de procureur du roi dans les deux compagnies et doit acquitter en 1712 une double taxation comme magistrat cumulant et membre de la maréchaussée ${ }^{22}$. L'aspect obligatoire de ce cumul prend sens à l'aune de la pensée juridique des $\mathrm{XVI}^{e}$ et $\mathrm{XVI} \mathrm{II}^{\mathrm{e}}$ siècles, encore prégnante dans la monarchie du siècle des Lumières $^{23}$. Dans une perspective romaniste, la possibilité légale d'exercer en même 
temps plusieurs fonctions royales découle de manière logique de la conception de l'administration perçue comme le prolongement subordonné de la juridiction. Ce cadre législatif et intellectuel rend non seulement compte de la légalité, mais aussi de la nécessité du cumul des offices de justice et de police. Dans les villes de Tulle et de La Rochelle, l'association imposée dénote, avec la présence de deux magistrats dans la maréchaussée, un réel contrôle exercé par les juges du présidial au-delà de la seule subordination réglementée par la législation monarchique. Toutefois, elle n'atteste pas pour autant de l'absorption totale de la maréchaussée par la sénéchaussée, perceptible dans le cas de Nantes ${ }^{24}$.

Les officiers «moyens » de judicature profitent de façon à la fois modérée et équilibrée des nouvelles charges vénales mises sur le marché dans la seconde moitié du règne de Louis XIV. Sur quarante-quatre possesseurs relevés, seulement douze détenteurs d'offices municipaux, de lieutenants généraux de police ou de subdélégués appartiennent à la magistrature seconde. La proportion obtenue de moins d'un tiers équivaut à peu près à leur représentation parmi l'ensemble des officiers cumulants. Elle suggère qu'ils n'ont ni bénéficié d'un appui privilégié de la monarchie cherchant à placer systématiquement des robins influents de la province dans les nouvelles fonctions, ni recherché avec empressement ces charges inédites. La concurrence de nouveaux venus a pu aussi contribuer à les évincer. En effet, dans plusieurs cas, les détenteurs montrent un investissement exclusif dans les récents offices vénaux, indicateur de leur engagement de frâiche date dans l'État royal: à Cognac, Jacques Perrin est ainsi maire en titre et lieutenant général de police; Pierre de Bonnet est maire à Ussel et à Clermont où il détient en sus la charge de subdélégué. La ventilation des officiers «moyens » de justice en possession d'un des trois types d'offices créés révèle par ailleurs une répartition équilibrée avec trois possesseurs d'un office de subdélégué, quatre d'une charge vénale municipale (en général de maire) et cinq de la lieutenance générale de la police. Un autre facteur explique sans doute leur place modeste dans les nouvelles formes de vénalité : le souci politique de l'administration monarchique de limiter la concentration excessive de l'autorité publique au profit d'un seul et même individu ${ }^{25}$. Cette probable préoccupation d'un équilibre local du pouvoir n'empêche pas en tout cas la constitution de quelques positions avantageuses dans les villes.

17 En effet, une partie des officiers «moyens» de judicature mobilise la nouvelle «marchandise d'État» afin de consolider leur situation personnelle et familiale dans la cité. Deux exemples empruntés aux présidiaux d'Auvergne incarnent les avantages procurés par le cumul multiple. À Aurillac, Amable Delort possède au début du XVIII siècle l'office de lieutenant général réuni à la charge de président. En devenant acquéreur en 1701 de la lieutenance générale de police pour 5000 livres et en 1704 de l'office de maire en titre, il renforce d'une place privilégiée dans les institutions urbaines sa position prééminente dans le tribunal, grâce à la détention conjointe des deux fonctions de chefs de la compagnie. Descendant d'une dynastie robine à la tête du siège depuis la première moitié du XVII siècle, bénéficiaire de fait d'une autorité locale indéniable, il possède dans le même temps un solide patrimoine officier, estimable pour les trois charges de justice et de police à 65000 livres $^{26}$. Le cumul des fonctions publiques représente pour Delort le fondement institutionnel et matériel d'une accumulation de pouvoir et d'influence sociale dans l'État et la cité. Il constitue aussi l'aboutissement des luttes de factions de la décennie 1690 pour contrôler le consulat et l'instrument pour la famille Delort d'une pérennisation de sa situation privilégiée dans le corps de ville durant le XVIII siècle $^{27}$. À 
Clermont, la famille Dufour détient de manière similaire une position forte dans le tribunal et la cité. Dans le présidial, Jean Dufour possède les charges de président et de lieutenant général, acquises respectivement en 1676 et en 1662 pour 28000 livres et 14 000 livres et son cousin David Dufour l'office de procureur du roi, acheté en 1696 pour 38 500 livres $^{28}$. Détentrice depuis la seconde moitié du XVII siècle des fonctions les plus coûteuses du siège, la dynastie Dufour renforce son assise locale par l'obtention de la lieutenance générale de police et sa réunion à la charge de lieutenant général. Pour ces deux familles auvergnates de magistrats présidiaux, la pluri-appartenance permet la possession concomitante et complémentaire de positions prééminentes dans l'appareil étatique local et sur la scène urbaine.

La répartition spatiale des officiers cumulants présentée dans le tableau 2 reflète de manière sommaire la hiérarchie urbaine des trois généralités ${ }^{29}$. Dans le Limousin et l'Auvergne, elle traduit de façon logique et comparable la primauté des villes présidiales qui regroupent respectivement trente-six et trente officiers en situation de cumul. De manière oblique, elle dénote leur statut de "villes d'Ancien Régime " ${ }^{30}$, définies par le rôle prépondérant des fonctions administratives. La corrélation établie en 1765 par l'intendant entre la dépopulation de Riom et la diminution de l'activité judiciaire illustre a contrario les liens entre la vitalité de l'administration et le rang urbain ${ }^{31}$. Cette distribution géographique montre aussi la distinction majeure entre les villes en position, au moins relative, de métropoles provinciales, mieux fournies en officiers cumulants (Limoges, Riom, La Rochelle) et les «capitales judiciaires secondaires $»^{32} \mathrm{~d}^{\prime} u n$ rang moindre, aux effectifs plus modestes (Angoulême, Tulle, Brive, Clermont, Aurillac). Le cas de la généralité de La Rochelle apparaît plus nuancé avec le regroupement de trente-huit officiers cumulants dans trois villes prépondérantes mais au profil distinct car $\mathrm{La}$ Rochelle est avant tout un grand port marchand tandis que la fonction militaire caractérise Rochefort et le modèle de la « ville d'Ancien Régime » s'applique à Saintes. 
Tableau 2. La répartition des officiers cumulants dans les villes des généralités de Limoges, Riom et La Rochelle (1709-1712)

\begin{tabular}{|l|l|l|l|l|l|l|l|l|}
\hline $\begin{array}{l}\text { Généralité } \\
\text { de Limoges }\end{array}$ & Limoges & Angoulême & Tulle & Brive & Uzerche & $\begin{array}{l}\text { Saint- } \\
\text { Yrieix } \\
\text { villes } \\
\text { (a) }\end{array}$ & Total \\
\hline nombre & 14 & 9 & 8 & 5 & 1 & 1 & 2 & 40 \\
\hline $\begin{array}{l}\text { généralité } \\
\text { de Riom }\end{array}$ & Riom & Clermont & Aurillac & Salers & $\begin{array}{l}\text { Saint- } \\
\text { Flour }\end{array}$ & Issoire & $\begin{array}{l}\text { petites } \\
\text { villes }\end{array}$ & Total \\
\hline nombre & 17 & 9 & 4 & 3 & 2 & 2 & 6 & 43 \\
\hline $\begin{array}{l}\text { généralité } \\
\text { de } \\
\text { Rochelle }\end{array}$ & La & Rochelle & Saintes & Rochefort & Cognac & $\begin{array}{l}\text { Saint-Jean } \\
\text { d'Angely }\end{array}$ & Maren- \\
nes & petites & Totlles \\
\hline nombre & 17 & 12 & 9 & 6 & 6 & 3 & 1 & 54 \\
\hline
\end{tabular}

a) : les petites villes comptent chacune un officier cumulant (Ussel et Bourganeuf dans la généralité de Limoges; Montferrand, Mauriac, Billom, Nouic, Nouette et Usson dans la généralité de Riom ; Brouage dans la généralité de La Rochelle).

19 La mise en perspective de la distribution de 1709-1712 avec une évaluation comptable des offices et des officiers «moyens » de la généralité de Riom dans la décennie 1720 montre qu'elle reflète en modèle réduit la répartition globale des compagnies intermédiaires. Le tableau IV dévoile en premier lieu la hiérarchie urbaine selon l'effectif des administrateurs de rang médian. Elle indique la prééminence attendue des trois villes présidiales de Riom, Clermont et Aurillac rassemblant $72 \%$ des officiers et $67 \%$ des offices «moyens » de la province ${ }^{33}$. La cité de Riom en compte à elle seule environ $30 \%$. Dans la capitale de l'Auvergne, ce groupe pèse d'ailleurs plus que le bureau des finances pourtant pourvu d'un personnel nombreux avec trente et un officiers. Cependant, le reflet apparaît partiel et quelque peu déformé. Si la corrélation est lisible entre la répartition spatiale des officiers cumulants et des officiers «moyens" pour le sommet de la hiérarchie urbaine, elle semble plus imparfaite pour les villes secondaires. Salers bénéficie ainsi d'un rang avantageux dans le premier classement alors que Murat en est absente en dépit de la localisation de deux compagnies intermédiaires. Le tableau IV révèle en second lieu le poids respectif des différents types d'officiers « moyens ». À l'échelle de la généralité, les officiers « moyens » de justice occupent une place prépondérante avec $68 \%$ du total (55\% pour les magistrats des présidiaux et $13 \%$ pour les juges des bailliages). Les officiers «moyens » de finance représentent seulement $28 \%$ de l'ensemble, dispersés au sein des sept élections. 


\section{La monarchie administrative et le cumul des fonctions publiques}

20 L'État royal a mis en place des normes anciennes et réitérées afin de réglementer la pratique répandue du cumul des fonctions publiques $^{34}$. En définissant avec clarté et précision deux types d'incompatibilité pour les offices royaux, la législation monarchique délimite le domaine du cumul toléré. Elle établit une différence majeure entre une incompatibilité relative concernant les offices royaux entre eux, notamment l'association de charges de justice avec des offices de finance grâce à des lettres de compatibilité et une incompatibilité absolue avec les offices seigneuriaux ou les activités commerciales même par personne interposée. Cette distinction nette entre une sphère de l'office royal où le cumul est possible, moyennant une autorisation explicite ou tacite, et la sphère des activités non royales où il est impossible a pour but de préserver l'indépendance et la dignité des serviteurs du roi. Elle proscrit de manière radicale l'exercice de fonctions entraînant soit la soumission à l'égard du seigneur, soit la motivation exclusive par le profit matériel. À la différence de la position intransigeante du parlement de Paris hostile à tout cumul des offices royaux ${ }^{35}$, la posture pragmatique de l'État royal définit des normes souples afin de l'encadrer. Néanmoins, les carrières officières simultanées posent parfois une ambiguïté essentielle à l'administration monarchique, contrainte d'accepter des situations de fait différentes de la pratique jugée idéale. Un exemple limousin et un cas auvergnat illustrent ses raisons mais aussi ses possibles réticences vis-à-vis du cumul toléré. En 1699, l'intendant de la généralité de Limoges Louis de Bernage justifie son avis favorable à Jean-Baptiste Jaucen, président du présidial et receveur ancien des tailles à Tulle (tableau I) :

Ces offices ne paraissent pas formellement incompatibles parce que ce sont des charges indépendantes l'une de l'autre et qui n'ont rien de connexes. Il ne peut y avoir qu'une incompatibilité de bienséance, n'étant pas convenable qu'un des chefs d'une Compagnie de Justice soit comptable et sujet aux contraintes [...]. Il peut être d'ailleurs détourné de l'application qu'il doit à ses offices par des fonctions si opposées [...]. Enfin, il serait mieux que cela ne fût pas. Cependant il était pourvu de tous les deux dès 1693 avant que je fusse Intendant de cette Province. On l'a toléré et on peut le tolérer encore si on voit que cet officier se comporte bien ${ }^{36}$.

21 L'intendant souligne deux inconvénients majeurs: le risque de l'affaiblissement de la dignité du magistrat par ses activités d'officier de finance, le danger latent de l'absentéisme dans son métier de juge. Toutefois, sa marge de manœuvre est limitée à la fois par la patrimonialité des offices vénaux et l'obligation de respecter la décision antérieure de son prédécesseur. La tolérance, mentionnée par Bernage ou indiquée par l'édit de 1709, doit se comprendre dans son acception ancienne et péjorative comme un pis-aller qu'il faut supporter. Confronté quelques années auparavant à une situation similaire, l'intendant de la généralité d'Auvergne approuve sans réserves le cumul d'un office d'avocat du roi au présidial d'Aurillac avec la charge de président de l'élection, en arguant de la fortune et de la considération sociale remarquables du demandeur ${ }^{37}$. En dépit de leur tonalité différente, les attitudes des deux représentants de la monarchie administrative suggèrent une raison commune mais inavouée de leur tolérance envers le cumul: sa nécessité sociale dans des villes de taille moyenne où l'élite administrative virtuelle se recrute dans un vivier réduit. 
Obligée d'accepter de facto ces pratiques cumulatives, l'administration royale les connaîtelles dans toute leur ampleur? Son information apparaît modeste et inégale à l'aune des maigres indications portant sur des officiers «moyens" dans les mémoires des intendants à l'extrême fin du xviI siècle. Dans la généralité de La Rochelle, le représentant du pouvoir central ne mentionne d'ailleurs aucun magistrat dans sa description des juridictions. Dans la province d'Auvergne, il note un seul cas de cumul imposé à Riom pour l'office couplé de procureur du roi du présidial et de la monnaie. Dans la généralité de Limoges, le commissaire départi relève six officiers cumulants ${ }^{38}$. Les rares mentions de carrières officières simultanées concernent uniquement des compatibilités jugées honorables, en excluant l'association plus contestable de charges de justice et de finance. Par leur faible nombre et leur profil sélectif, elles contrastent avec l'importance et la diversité des cumuls relevés dans les nomenclatures. Cette dissymétrie flagrante n'indique pas obligatoirement un manque crucial d'informations pour la monarchie administrative à la fin du XVII siècle. Elle atteste plus vraisemblablement du souci politique des intendants de dissimuler les pratiques cumulatives les moins acceptables afin de ménager les intérêts des élites officières provinciales et de jeter un voile pudique sur leur politique de tolérance et de compromis. Lorsque la préoccupation fiscale prédomine en 1709, ils peuvent et doivent mobiliser les renseignements sciemment occultés auparavant. L'intendant de la généralité de La Rochelle dévoile alors la panoplie des tactiques mobilisées par les officiers royaux pour contourner le cumul désormais fiscalisé. Il recense trois parades: la technique vénale de distinction entre titulaire et propriétaire de l'office, l'exercice de fonctions publiques par commission et la vente anticipée des offices pour mettre fin au cumul. À La Rochelle, Valentin Mariocheau utilise le premier subterfuge car il possède la charge de subdélégué mais son office de lieutenant général appartient à la dame Bernardin ; à Saint-Jean d'Angely, Alphonse Lebat emploie le second moyen pour exercer la recette ancienne et alternative des tailles; à Rochefort, Jean-René Lardreau recourt au troisième biais en vendant ses offices de procureur du roi des eaux et forêts et de subdélégué. Soucieuse de valider ou d'infirmer les justifications de ces officiers demandant une décharge, l'administration royale sait alors mobiliser une information précise et fiable sur l'ampleur réelle des pratiques cumulatives.

Dans les trois généralités de Limoges, Riom et La Rochelle, la politique de fiscalisation du cumul en 1709 représente un échec patent pour la monarchie administrative. Elle se fonde sur le principe simple d'une imposition proportionnelle à la valeur additionnée des charges, selon une répartition des taxes en six catégories ${ }^{39}$. En sollicitant les finances de ses élites officières, la monarchie de Louis XIV mobilise une nouvelle fois leur crédit privé au service du crédit d'État ${ }^{40}$. En confirmant selon les termes de l'acte royal de mars 1709 un privilège obtenu par édit, lettre de compatibilité ou approprié de fait par tolérance, elle applique une politique classique de distribution sélective du privilège contre une compensation monétaire. Dans les provinces du Limousin, de l'Auvergne et de l'AunisSaintonge, la mesure fiscale ne rapporte pas les fonds escomptés. Il reste à recouvrer 78 110 livres sur les 103500 livres prévues dans la généralité de Limoges en mars 1712 et 128 700 livres sur une somme globale estimable à 140000 livres pour la généralité de Riom en septembre $1713^{41}$. L'échec de l'État royal à fiscaliser le cumul résulte de l'absence majoritaire de paiement par les officiers, repérable pour la moitié d'entre eux en Limousin, les trois-quarts en Auvergne et la quasi-totalité en Saintonge et en Aunis. Confrontée aux plus fortes réticences dans cette dernière généralité, l'administration doit 
même recourir à la contrainte financière. Elle utilise des huissiers extérieurs aux villes concernées afin de contourner les pressions sociales exercées sur les auxiliaires de justice du lieu par les notables urbains sujets à la taxation. Le paiement minoritaire apparaît parfois fort tardif, s'étalant même jusqu'en 1717 pour un officier auvergnat ${ }^{42}$. Dans d'autres provinces du royaume, les oppositions similaires des officiers entraînent en 1712 une situation identique, marquée par la modicité des rentrées financières. Dans la généralité de Caen, il reste à recouvrer 124891 livres sur les 133650 livres prévues ; dans la généralité de Moulins, 49357 livres demeurent impayées sur les 58080 livres exigées après modération ${ }^{43}$. Cet échec généralisé de la fiscalisation du cumul des offices royaux s'inscrit dans la crise du «système fisco-financier» à la fin du règne de Louis XIV, principalement définie par le rendement déclinant des affaires extraordinaires.

Quelles raisons expliquent les réticences d'une majorité d'officiers royaux à acquitter la taxe sur le cumul ? L'administration monarchique suggère des interprétations probantes en distinguant motivations économiques et politiques. En 1713, l'intendant des finances de Bercy établit une différence entre les officiers cumulants pouvant bénéficier d'une possible modération, « ceux qui sont dans l'impuissance de payer entièrement la taxe » et leurs confrères aussi en situation de non-paiement mais sans justification valable, « ceux qui sont connus pour être riches $\aleph^{44}$. Il recommande d'appliquer une politique de compromis dans le premier cas et d'intransigeance dans le second cas, avec la saisie éventuelle des gages. Cette incitation officielle à vérifier l'existence de deux attitudes officières distinctes constitue aussi une invitation heuristique à approfondir le poids respectif des deux types de motivations.

La faiblesse économique des officiers royaux représente très vraisemblablement une explication secondaire de l'absence dominante de paiement. Dans la généralité de La Rochelle, seul Jean Couppeau, greffier de police, greffier ancien de l'hôtel de ville et contrôleur du greffe à La Rochelle peut en être exempté selon l'intendant comme " chargé d'une nombreuse famille qu'il a de la peine à faire subsister ». Dans la généralité de Limoges, la mise en relation du faible empressement de deux magistrats présidiaux à acquitter leur imposition et de leurs solides fortunes montre l'aspect second de la motivation financière. À Angoulême, le lieutenant général et maire en titre de la ville, Chirade, verse seulement 46 livres sur les 5400 livres exigées. Or, à la même époque, il est inclus avec d'autres juges du siège dans un «état des gens les plus aisés de ce département » en raison de ses importants revenus :

[...] le lieutenant général d'Angoulême a au moins 50000 livres de rente, et 150000 livres au moins sur la place à Paris, et ne fait aucune dépense : je l'ai compris dans mon état pour 20000 livres, parce ce que je suis persuadé qu'il payera cette somme aussi aisément qu'aucun homme de France ${ }^{45}$.

Détenteur d'un patrimoine conséquent, possesseur d'un crédit financier considérable, il apparait tout à fait apte à acquitter les différentes taxes étatiques. À Limoges, le conseiller et trésorier de France Jean-François Martin de la Bastide, qui n’a pas réglé les 2700 livres demandées, est, lui aussi, en possession d'une fortune immobilière et mobilière solide, estimable pour la seconde composante à environ 80000 livres $^{46}$. Pour ces deux magistrats, le paiement parcimonieux ou inexistant de la taxe de 1709 demeure déconnecté de la question de leur capacité financière.

L'explication essentielle des réticences des officiers royaux réside dans leur mécontentement vis-à-vis de la politique vénale intensive menée dans la seconde moitié du règne de Louis XIV. Confrontés à des augmentations successives de gages - notamment 
les magistrats des présidiaux en 1689 et 1693 - et à des créations de nouveaux offices parfois acquis de manière imposée par les compagnies ${ }^{47}$, ils rechignent à payer la taxation supplémentaire de 1709. En Aunis et en Saintonge, plusieurs d'entre eux font allusion à la technique vénale d'unir dans un "même corps d'offices » plusieurs charges afin de justifier leur absence du paiement de la taxe. Jean Poirel mentionne ainsi à La Rochelle le cumul imposé de l'office de conseiller au présidial, «réuni forcé par arrêt du conseil » avec celui d'assesseur de la maréchaussée. Le contexte difficile des années 1709-1712, marqué par le «Grand Hiver » et la création de l'impôt universel du dixième en 1710, renforce leur mécontentement vis-à-vis de l'accumulation des taxations en engendrant une disette conjoncturelle d'argent qui empêche les élites officières de se procurer avec facilité les liquidités exigées. C'est pourquoi la monarchie administrative est contrainte de revoir à la baisse ses prétentions financières. Dans la généralité de La Rochelle, l'intendant propose un nombre important de modérations, touchant vingt et un officiers sur quarante-deux concernés et de grande ampleur puisqu'elles divisent par deux ou par trois les taxes initiales. Coincé entre les réticences d'une majorité de ses serviteurs et une conjoncture économique particulièrement défavorable, l'État royal doit adopter des attentes fiscales plus modestes.

\section{Esquisse d'une typologie des officiers « moyens » cumulants}

Envisagées dans la perspective des motivations des officiers «moyens", les carrières simultanées correspondent à trois types: le cumul logique, le cumul économique et le cumul stratégique. Fondé sur la notion de compétence, le premier type traduit le souci d'une captation des nouvelles charges afin de préserver ou d'augmenter ses prérogatives. Lié à la recherche de la rentabilité, le second type manifeste la volonté d'accroître ses revenus par une participation diversifiée à l'État d'offices. Engendré par la préoccupation de l'honorabilité, le troisième type vise à obtenir un statut plus avantageux dans la société. Ancrée dans les multiples valeurs du monde de l'office, cette typologie ternaire des pratiques cumulatives en ordonne la diversité.

Chez les officiers «moyens", le cumul logique prend des formes simples. Il correspond pour les officiers de finance à la possession des différentes recettes de taille d'une même élection, pour les officiers de justice à la détention concomitante des charges de président présidial et de lieutenant général ou à l'appropriation de la lieutenance générale de police. Il s'agit soit de conserver les compétences de sa charge originelle (et ses revenus pour les receveurs), soit de les accroître. Dans les sièges de Saintes, Clermont et Riom, le cumul des offices de chefs de la compagnie règle, au moins provisoirement, le délicat problème institutionnel de l'équilibre du tribunal siamois, avec une sénéchaussée dirigée par le lieutenant général et un présidial sous la direction du président ${ }^{48}$. L'acquisition de l'office de lieutenant général de police revêt des significations différentes selon l'autorité en charge de la police urbaine avant 1699. À Limoges, elle est synonyme de la préservation des compétences intégrales de sa charge pour le lieutenant général Rogier des Essarts déjà détenteur d'une position prééminente et acquéreur de la nouvelle fonction en 1701 pour 18000 livres. À Angoulême, elle permet au président de Bouet une augmentation essentielle de ses prérogatives au détriment du maire. Dans la cité de l'Angoumois, elle renforce aussi le patrimoine officier du magistrat : acquise pour 22000 
livres, la lieutenance générale de police équivaut à la charge de président revendue vers 1720 pour 24000 livres $^{49}$.

30 Le cumul économique représente en priorité un instrument d'accumulation financière grâce aux offices royaux, envisagés comme des placements rentables. Perceptible dans le monde robin en général, la prépondérance accordée aux motivations matérielles apparaitt aussi repérable chez des officiers subalternes ${ }^{50}$. Pour les officiers « moyens ", la détention simultanée de charges de receveurs des tailles incarne l'idéal-type de «l'investissement cumulatif $^{51}$. Toutefois, la possession concomitante d'offices secondaires ou atypiques peut être aussi lucrative au vu de deux cas auvergnats. À Clermont, l'élu Daniel Chamaret possède en 1709 la charge de greffier en chef de l'élection qui lui rapporte 455 livres de gages et 400 livres d'émoluments par an. Seconde dans la hiérarchie officière, cette fonction produit néanmoins des revenus supérieurs à l'office de finance et sa valeur de 18 000 livres la place au-dessus de la charge d'élu alors estimable à 15000 livres $^{52}$. À Riom, le conseiller chevalier d'honneur du présidial Jean-Paul Courtin détient aussi l'office de trésorier de France au bureau des finances, acquis en 1692 et la charge de commissaire de la marine, couplée avec la détention de trois offices d'archers de la marine. Si sa première fonction lui procure la considération sociale liée à l'exercice de la justice royale, ses autres modalités d'insertion dans l'État royal lui garantissent des revenus importants : 2 495 livres par an pour l'office de finance, 4400 livres annuelles pour ses diverses charges dans la marine. L' « investissement cumulatif » de Courtin correspond à une participation simultanée aux dimensions judiciaire, financière et administrative de la monarchie. Traduisant une forte diversification des placements et la recherche patente de leur rentabilité, il résulte aussi d'un engagement précoce et durable de la famille Courtin dans des fonctions secondaires mais lucratives. En effet, la possession au début du XVII siècle par son grand-père Antoine du greffe ancien du bureau des finances puis alternatif et triennal avec son père Georges jusqu'à leur vente en 1711 pour 50000 livres, a permis un fructueux enrichissement sur presque un siècle et le glissement vers des formes plus élevées de cumul économique ${ }^{53}$. Modeste pour Daniel Chamaret, considérable pour JeanPaul Courtin, l'accumulation financière en capital et en revenus motive leur intégration diversifiée à l'État d'offices.

31 Le troisième type de cumul qualifié de stratégique traduit la recherche prépondérante de l'honorabilité. Dans les généralités de Limoges, Riom et La Rochelle, l'acquisition des offices anoblissants des bureaux des finances représente la voie possible d'un anoblissement certes graduel mais sur place que choisissent trois officiers «moyens » de judicature. À Riom, le conseiller présidial Claude de Chardon des Roys et de Poujol obtient en 1700 la charge de trésorier de France. Dans des circonstances familiales défavorables, marquées par la disparition sans descendance masculine de son frère aîné et détenteur de la charge, la transmission a pour but de préserver le patrimoine paternel et d'assurer une continuité dynastique ${ }^{54}$. Dans le cadre d'un anoblissement fondé sur la détention de l'office sur deux générations pendant vingt ans pour chacune, le souci de le maintenir dans la famille est logique. Il représente le pari d'acquérir la noblesse sur la durée mais il comporte une importante dimension aléatoire, en regard du faible nombre de familles auvergnates bénéficiaires d'une authentique promotion ${ }^{55}$. Pour la dynastie de Chardon, l'accession à l'honorabilité semble avérée puisque Claude transmet l'office en 1740 à son fils Pierre. En Auvergne, le faible nombre d'officiers «moyens » de judicature détenteurs simultanément d'un office anoblissant du bureau des finances et leur absence de détention de charges de conseillers de la Cour des aides n'indiquent pas de glissement 
massif vers les fonctions les plus attractives de l'État de finance, garantes de revenus et de privilèges conséquent ${ }^{56}$. Prédominante, l'honorabilité ne constitue toutefois pas l'unique motivation de ces officiers cumulants ; l'intérêt économique d'un investissement rentable est une raison complémentaire, en comparaison de la valeur modérée des offices de justice ${ }^{57}$. Dans le cas de Claude de Chardon, sa charge de trésorier de France, échangée à hauteur de 18 000-22 000 livres dans la décennie 1710 vaut deux fois plus que son office de conseiller garde-scel, estimé alors à $10000-11000$ livres $^{58}$. À Limoges, la disproportion apparaît encore plus conséquente à la même période: les charges de conseillers du présidial se vendent environ 8000 livres tandis que les offices de trésoriers de France atteignent les 30000 livres.

Le dénombrement des officiers cumulants au début du XVIII siècle offre un utile bilan statistique pour évaluer la place des officiers « moyens » au sein de l'État royal, constitue un indicateur ponctuel de l'état de leurs relations avec la monarchie administrative et représente un révélateur pertinent de pratiques officières souvent peu perceptibles. Les recensements initiés en 1709 des officiers cumulants montre, en particulier dans les généralités de Limoges et de Riom, à la fois la surreprésentation numérique des officiers «moyens ", notamment de judicature parmi les serviteurs du roi en situation de cumul et leur remarquable ubiquité au sein des institutions monarchiques. Attestant de fréquentes insertions simultanées et plurielles dans l'État d'offices, ils témoignent à l'orée du siècle des Lumières de la permanence d'une élite administrative polymorphe, pourvue de compétences génériques et soucieuse d'un investissement étatique diversifié. Instruments au service de la "vénalité légale ", ils révèlent aussi la cristallisation de profondes divergences entre la monarchie administrative et les officiers au début du XVIII siècle. Si le cumul apparait souvent toléré par l'État royal, sa fiscalisation inédite en 1709 se solde par un échec financier généralisé dans les trois généralités de Limoges, Riom et La Rochelle. Les réticences d'une large majorité d'officiers "moyens» à acquitter la nouvelle taxation témoigne avant tout de leur mécontentement croissant vis-à-vis de la politique vénale intensive menée dans la seconde moitié du règne de Louis XIV. Elles contribuent à aggraver, particulièrement pour les magistrats présidiaux, un contentieux engendré par l'accumulation d'augmentations de gages et de créations d'offices depuis la décennie 1680 .

Le dénombrement constitue enfin l'opportunité de dévoiler et d'appréhender des comportements officiers généralement peu perceptibles. Les nomenclatures de la fin du règne de Louis XIV montrent la diversité des pratiques cumulatives des officiers « moyens ». Une typologie envisageant leurs significations professionnelles et sociales les ordonne en trois catégories. Le cumul logique traduit le souci prioritaire de conserver ou d'accroître ses compétences en acquérant des charges de nature similaire ou proche. Le cumul économique correspond à la recherche prépondérante de la rentabilité des offices, notamment de finance et repose souvent sur le contournement toléré ou dissimulé des normes officielles définissant les incompatibilités de fonctions publiques. Le cumul stratégique manifeste une attention prépondérante pour l'honorabilité des charges, en particulier anoblissantes. Au-delà de ses visées initiales, le dénombrement administratif des officiers royaux cumulants lève ainsi le voile sur des pratiques officières souvent occultées. 
Tableau I. Les magistrats cumulants des présidiaux de la généralité de Limoges en 1712

\begin{tabular}{|c|c|c|c|c|c|}
\hline Présidial & $\begin{array}{ll}\text { Noms des } \\
\text { magistrats }\end{array}$ & Offices cumulés & $\begin{array}{l}\text { Nombre } \\
\text { d'offices } \\
\text { cumulés }\end{array}$ & $\begin{array}{l}\text { Montant de } \\
\text { la taxation } \\
\text { (en livres) }\end{array}$ & $\begin{array}{l}\text { Montant } \\
\text { payé en } \\
1712 \quad \text { en } \\
\text { livres) }\end{array}$ \\
\hline & $\begin{array}{ll}\text { Pierre } & \text { du } \\
\text { Verdier } & \text { de } \\
\text { Genouilhac } & \end{array}$ & $\begin{array}{l}* \text { conseiller } \\
* \text { secrétaire du roi }\end{array}$ & 2 & 1800 & 0 \\
\hline \multirow[t]{3}{*}{ Brive } & $\begin{array}{l}\text { Jean-Baptiste } \\
\text { Noiret de la } \\
\text { Garde }\end{array}$ & $\begin{array}{l}\text { * conseiller } \\
* \text { secrétaire du roi }\end{array}$ & 2 & 1800 & 0 \\
\hline & $\begin{array}{l}\text { Dominique } \\
\text { Dubois }\end{array}$ & $\begin{array}{l}\text { * conseiller } \\
\text { * élu }\end{array}$ & 2 & 900 & 0 \\
\hline & $\begin{array}{l}\text { Joseph Rogier } \\
\text { des Essarts }\end{array}$ & $\begin{array}{ll}* & \text { lieutenant } \\
\text { général } & \\
* & \text { lieutenant } \\
\text { général de police } \\
* & \text { lieutenant de } \\
\text { maire } & \\
* & \text { auditeur des } \\
\text { comptes }\end{array}$ & 4 & 5400 & 1800 \\
\hline \multirow[t]{4}{*}{ Limoges } & $\begin{array}{l}\text { Jean Vidaud du } \\
\text { Dognon }\end{array}$ & \begin{tabular}{|lr}
$*$ & lieutenant \\
particulier \\
$*$ & lieutenant \\
général d'épée
\end{tabular} & 2 & 1800 & 1800 \\
\hline & $\begin{array}{l}\text { Jacques de } \\
\text { Douhet }\end{array}$ & $\begin{array}{l}* \quad \text { lieutenant } \\
\text { criminel } \\
* \text { juge-garde de la } \\
\text { Monnaie }\end{array}$ & 2 & 1800 & 0 \\
\hline & $\begin{array}{l}\text { Jean-François } \\
\text { Martin de la } \\
\text { Bastide }\end{array}$ & \begin{tabular}{|l}
$*$ conseiller \\
$* \quad$ Trésorier de \\
France \\
$* \quad$ commissaire \\
enquêteur
\end{tabular} & 3 & 2700 & 0 \\
\hline & $\begin{array}{l}\text { Jean-Baptiste } \\
\text { Jaucen de } \\
\text { Poissac }\end{array}$ & $\begin{array}{l}* \text { président } \\
\text { * receveur ancien } \\
\text { des tailles }\end{array}$ & 2 & 5400 & 0 \\
\hline
\end{tabular}




\begin{tabular}{|c|c|c|c|c|c|}
\hline & $\begin{array}{ll}\text { Jean-Martial } & \text { de } \\
\text { Fénis } & \text { de } \\
\text { Lacombe } & \end{array}$ & $\begin{array}{l}\text { * procureur du roi } \\
\text { * procureur du roi } \\
\text { de la ville } \\
\text { * procureur du roi } \\
\text { de } \\
\text { maréchaussée }\end{array}$ & 3 & 1800 & 1800 \\
\hline & $\begin{array}{l}\text { Ignace Darluc- } \\
\text { Delpy }\end{array}$ & $\begin{array}{l}\text { * conseiller } \\
\text { * élu }\end{array}$ & 2 & 900 & 900 \\
\hline \multirow[t]{5}{*}{ Tulle } & $\begin{array}{l}\text { François- } \\
\text { Martial Melon }\end{array}$ & $\begin{array}{l}\text { * avocat du roi } \\
* \quad \text { receveur des } \\
\text { décimes }\end{array}$ & 2 & 2700 & 0 \\
\hline & $\begin{array}{ll}\text { Gabriel } & \text { du } \\
\text { Myrat } & \text { de } \\
\text { Boussac } & \end{array}$ & $\begin{array}{l}\text { * conseiller } \\
\text { d'honneur } \\
* \text { contrôleur des } \\
\text { décimes }\end{array}$ & 2 & 450 & 0 \\
\hline & $\begin{array}{l}\text { Léger de } \\
\text { Braquilanges }\end{array}$ & $\begin{array}{l}\text { * conseiller } \\
\text { * assesseur à la } \\
\text { maréchaussée }\end{array}$ & 2 & 450 & 0 \\
\hline & Chirade & $\begin{array}{l}* \quad \text { lieutenant } \\
\text { général } \\
* \text { maire ancien }\end{array}$ & 2 & 5400 & 46 \\
\hline & $\begin{array}{l}\text { Arnaud de } \\
\text { Bouet }\end{array}$ & $\begin{array}{l}\text { * président } \\
* \quad \text { lieutenant } \\
\text { général de police }\end{array}$ & 2 & 2700 & 0 \\
\hline Angoulême & $\begin{array}{l}\text { Barreau de } \\
\text { Girac }\end{array}$ & $\begin{array}{l}\text { * procureur du roi } \\
\text { * procureur du roi } \\
\text { de police }\end{array}$ & 2 & 2700 & 0 \\
\hline & Desruaux & $\begin{array}{l}\text { * conseiller } \\
\text { honoraire } \\
\text { * subdélégué }\end{array}$ & 2 & 450 & 0 \\
\hline & $\begin{array}{l}\text { Pasquier de } \\
\text { Pusgou }\end{array}$ & $\begin{array}{l}\text { * conseiller } \\
\text { * conseiller en la } \\
\text { maréchaussée }\end{array}$ & 2 & 900 & 0 \\
\hline
\end{tabular}

Source : Arch. nat., $H^{1} 1588$ 29c , p. 7 : «Estat des recouvrements et des sommes payés par Messieurs les officiers compris aux rolles arestez au Conseil pour la compatibilité en consequence de l'Edit de mars $1709 »$-du 12 mars 1712. 
Tableau II. Les magistrats cumulants des présidiaux de la généralité de Riom en 1709

\begin{tabular}{|c|c|c|c|c|c|}
\hline Présidial & $\begin{array}{l}\text { Noms des } \\
\text { magistrats }\end{array}$ & Offices cumulés & $\begin{array}{l}\text { Nombre } \\
\text { d'offices } \\
\text { cumulés }\end{array}$ & $\begin{array}{l}\text { Montant de } \\
\text { la taxation } \\
\text { (en livres) }\end{array}$ & $\begin{array}{l}\text { Montant } \\
\text { payé en } 1713 \\
\text { (en livres) }\end{array}$ \\
\hline & $\begin{array}{l}\text { Gabriel- } \\
\text { Charles de } \\
\text { Combes }\end{array}$ & $\begin{array}{l}* \text { président } \\
* \text { lieutenant général } \\
* \quad \text { commissaire } \\
\text { enquêteur }\end{array}$ & 3 & 5400 & 0 \\
\hline & $\begin{array}{l}\text { Guillaume de } \\
\text { Vaux }\end{array}$ & $\begin{array}{l}\text { * procureur du roi } \\
\text { * procureur du roi } \\
\text { de la maréchaussée } \\
\text { * procureur du roi } \\
\text { de la monnaie }\end{array}$ & 3 & 5400 & 0 \\
\hline & $\begin{array}{l}\text { Claude de } \\
\text { Chardon }\end{array}$ & $\begin{array}{l}* \text { conseiller garde- } \\
\text { scel } \\
* \quad \text { Trésorier de } \\
\text { France }\end{array}$ & 2 & 3600 & 0 \\
\hline \multirow[t]{3}{*}{ Riom } & $\begin{array}{l}\text { Amable- } \\
\text { Jacques } \\
\text { Soubrany }\end{array}$ & $\begin{array}{l}\text { * conseiller } \\
* \text { maître particulier } \\
\text { des eaux et forêts }\end{array}$ & 2 & 2700 & 0 \\
\hline & $\begin{array}{c}\text { Amable } \\
\text { Vernaison }\end{array}$ & $\begin{array}{l}* \text { conseiller } \\
* \quad \text { lieutenant } \\
\text { particulier des eaux } \\
\text { et forêts }\end{array}$ & 2 & 2700 & 0 \\
\hline & Amable Rollet & $\begin{array}{l}* \text { conseiller } \\
* \text { secrétaire en la } \\
\text { chancellerie de la } \\
\text { Cour des aides }\end{array}$ & 2 & 2700 & 0 \\
\hline & Jean Dufour & $\begin{array}{l}\text { * président } \\
\text { * lieutenant général } \\
* \text { lieutenant général } \\
\text { de police }\end{array}$ & 3 & 5400 & 0 \\
\hline & David Dufour & $\begin{array}{l}\text { * procureur du roi } \\
\text { * procureur du roi } \\
\text { de la maréchaussée }\end{array}$ & 2 & $?$ & $\begin{array}{l}\text { paiement } \\
\text { effectué }\end{array}$ \\
\hline Clermont & Jean Redon & $\begin{array}{l}* \text { conseiller } \\
* \quad \text { Trésorier de } \\
\text { France (Riom) }\end{array}$ & 2 & 2700 & 0 \\
\hline
\end{tabular}




\begin{tabular}{|l|l|l|l|l|l|}
\hline & $\begin{array}{l}\text { Jean-Baptiste } \\
\text { Vachier }\end{array}$ & $\begin{array}{l}* \text { conseiller } \\
* \text { conseiller garde- } \\
\text { scel }\end{array}$ & 2 & 1800 & 0 \\
\hline Aurillac & Amable Delort & $\begin{array}{l}* \text { lieutenant général } \\
\text { enquêteur } \\
* \text { maire }\end{array}$ & 3 & 3600 & 0 \\
\hline & $\begin{array}{l}\text { Jean-Baptiste } \\
\text { Trenty }\end{array}$ & $\begin{array}{l}* \text { procureur du roi } \\
\text { procureur du roi } \\
\text { de la maréchaussée }\end{array}$ & 2 & 2700 & 0 \\
\hline
\end{tabular}

Sources : Arch. dép. Puy-de-Dôme, 1 C 4856 : « Etat des officiers sujets à l'edit de mars 1709 » [25 juin 1709] ; Arch. dép. Puy-de-Dôme, 1 C 4862 : «Estat des particuliers employes dans les rolles lesquels n'ont point entrer en payement » [septembre 1713].

Tableau III. Les magistrats cumulants des présidiaux de la généralité de La Rochelle en 1712

\begin{tabular}{|c|c|c|c|c|c|}
\hline Présidial & $\begin{array}{ll}\text { Noms } & \text { des } \\
\text { magistrats } & \end{array}$ & Offices cumulés & $\begin{array}{l}\text { Nombre } \\
\text { d'offices } \\
\text { cumulés }\end{array}$ & $\begin{array}{l}\text { Montant de } \\
\text { la taxation } \\
\text { (en livres) }\end{array}$ & $\begin{array}{l}\text { Montant } \\
\text { payé en } 1712 \\
\text { (en livres) }\end{array}$ \\
\hline & $\begin{array}{l}\text { Valentin } \\
\text { Mariocheau de } \\
\text { Bonnemour }\end{array}$ & $\begin{array}{l}* \text { lieutenant } \\
\text { général } \\
\text { * subdélégué } \\
\text { *commissaire } \\
\text { enquêteur } \\
\text { examinateur }\end{array}$ & 3 & 3600 & 0 \\
\hline & $\begin{array}{l}\text { Simon } \\
\text { Bouchereau }\end{array}$ & $\begin{array}{l}\text { * procureur du roi } \\
\text { * procureur du roi } \\
\text { de l'hôtel de ville } \\
\text { * procureur du roi } \\
\text { de la maréchaussée }\end{array}$ & 3 & 1800 & 0 \\
\hline \multirow[t]{2}{*}{$\begin{array}{l}\text { La } \\
\text { Rochelle }\end{array}$} & Jean Poirel & $\begin{array}{l}\text { * conseiller } \\
\text { * assesseur de la } \\
\text { maréchaussée }\end{array}$ & 2 & 450 & 50 \\
\hline & Serrat & $\begin{array}{l}\text { * conseiller garde- } \\
\text { scel } \\
* \text { lieutenant de la } \\
\text { maréchaussée } \\
\text { (Marennes) } \\
* \quad \text { commissaire } \\
\text { d'artillerie }\end{array}$ & 3 & 450 & 0 \\
\hline
\end{tabular}




\begin{tabular}{|l|l|l|l|l|l|}
\hline Saintes & Blaise de Gascq & $\begin{array}{l}* \text { président } \\
* \text { lieutenant } \\
\text { général lieutenant } \\
* \quad 4 \\
\text { général de police } \\
\text { * subdélégué }\end{array}$ & 5400 & 0 \\
\hline & De Beaune & $\begin{array}{l}\text { * procureur du roi } \\
\text { * procureur du roi } \\
\text { de police }\end{array}$ & 2 & 900 & 0 \\
\hline
\end{tabular}

Source : Arch. nat., K 891, n 51 (fol. 9-15) : état des offices de la généralité de La Rochelle du 30 avril 1712 par rapport à l'édit de compatibilité de mars 1709.

Tableau IV. Les offices et les officiers « moyens » dans les villes de la généralité de Riom en 1723-1725

\begin{tabular}{|c|c|c|c|c|}
\hline Ville & institution & $\begin{array}{l}\text { nombre des } \\
\text { offices }\end{array}$ & $\begin{array}{l}\text { nombre des } \\
\text { offices vacants }\end{array}$ & $\begin{array}{l}\text { nombre des officiers } \\
\text { en fonction }\end{array}$ \\
\hline & présidial & 31 & 1 & 29 \\
\hline & élection & 7 & 0 & 5 \\
\hline \multirow[t]{4}{*}{ Riom } & $\begin{array}{l}\text { maîtrise des eaux } \\
\text { et forêts }\end{array}$ & 3 & 0 & 3 \\
\hline & grenier à sel & 3 & 1 & 2 \\
\hline & total & 44 & 2 & 39 \\
\hline & présidial & 25 & 2 & 23 \\
\hline \multirow[t]{3}{*}{ Clermont } & élection & 7 & 0 & 7 \\
\hline & total & 32 & 2 & 30 \\
\hline & présidial & 27 & 5 & 22 \\
\hline \multirow[t]{3}{*}{ Aurillac } & élection & 7 & 0 & 5 \\
\hline & total & 34 & 5 & 27 \\
\hline & bailliage & 9 & 3 & 5 \\
\hline \multirow[t]{3}{*}{ Saint-Flour } & élection & 8 & 0 & 6 \\
\hline & total & 17 & 3 & 11 \\
\hline & $\begin{array}{l}\text { bailliage } \\
\text { (d'Andelat) }\end{array}$ & 3 & 0 & 3 \\
\hline
\end{tabular}




\begin{tabular}{|l|l|l|l|l|}
\hline Murat & $\begin{array}{l}\text { maitrise des eaux } \\
\text { et forêts }\end{array}$ & 3 & 0 & 0 \\
\hline & total & 6 & 0 & 3 \\
\hline Salers & bailliage & 5 & 0 & 5 \\
\hline Montferrand & bailliage & 7 & 2 & 4 \\
\hline \hline Issoire & élection & 7 & 0 & 6 \\
\hline Brioude & élection & 7 & 0 & 6 \\
\hline Mauriac & élection & 4 & 0 & 2 \\
\hline Total global & & $\mathbf{1 6 3}$ & $\mathbf{1 4}$ & $\mathbf{1 3 3}$ \\
\hline
\end{tabular}

Sources : Arch. dép. Puy-de-Dôme, 1 C 7065 : «Etat des offices royaux qui existent actuellement en la generalité de Riom et province d'Auvergne », «Etat de la situation actuelle des juridictions royales de la generalité de Riom et province d'Auvergne [1723-1725] ; Arch. dép. Puy-de-Dôme, 1 C 7066 (état du présidial d'Aurillac de 1724) ; Arch. dép. Puy-de-Dôme, 1 C 7068 (note sur le bailliage de Montferrand 1723-1725).

34 Afin de faciliter les comparaisons, les offices de receveurs des tailles sont toujours englobés avec ceux de l'élection stricto sensu.

\section{NOTES}

1. William Doyle, « Colbert et les offices », Histoire, Économie, Société, n 4, 2000, p. 469-480.

2. Pierre Chaunu a tenté naguère de quantifier la « technostructure » de l'État royal entre 1515 et 1665, notamment grâce à des pesées globales de l'État de justice et de l'État d'offices in Pierre Chaunu, Richard Gascon, sd., Histoire économique et sociale de la France.1 De 1450 à 1660 : l'État et la Ville, Paris, PUF, 1977, p. 126-127 et 197-200 ; René Favier, Les villes du Dauphiné aux XVII et XVIII siècles, Grenoble, Presses de l'Université de Grenoble, 1993, p. 270-275 propose une évaluation du personnel administratif et judiciaire des villes de Grenoble, Valence et Vienne à la fin du XVIII siècle.

3. Jean Nagle, «Les officiers «moyens" français dans les enquêtes sur les offices (XVI ${ }^{\mathrm{e}}-\mathrm{XVIII}{ }^{\mathrm{e}}$ siècles) », p. 25-41, Les officiers "moyens" à l'époque moderne: pouvoir, culture, identité, Michel Cassan (sd.), Limoges, Pulim, 1998.

4. Simone Meyssonier, La Balance et l'Horloge. La Genèse de la pensée libérale en France en XVIII siècle, Paris, Éditions de la Passion, 1989, p. 35-51.

5. Arch. nat., $\mathrm{H}^{1} 1588^{44}$ : documents relatifs aux affaires extraordinaires de la généralité de Rouen (1700-1712). En 1711, Boisguilbert paie 5000 livres d'augmentations de gages pour ses deux offices de président, sans doute acquis pour 4000 et 3500 livres après modération ( $f^{\circ} .27$ et 31 : 
tableau sur la création d'un office de second président des présidiaux en février 1705 et recouvrement forcé du 25 septembre 1711).

6. Ibid., état du recouvrement forcé du 25 septembre 1711 en vertu de l'édit de mars 1709 indiquant sa taxation pour 5400 livres avec l'avis suivant : «[Boisguilbert] a demandé qu'en payant 6000 livres par an on le laissât en repos, assez zélé ».

7. Robert Descimon, «Les élites du pouvoir et le prince : l'État comme entreprise ", Les élites du pouvoir et la construction de l'État en Europe, Wolfgang Reinhard (sd.), Paris, PUF, 1996 (p. 133-162).

8. Robert Descimon, Jean-Frédéric Schaub, Bernard Vincent (éds), Les Figures de l'administrateur. Institutions, réseaux et pouvoirs en Espagne, en France et au Portugal, XVI ${ }^{e}$-XIX ${ }^{e}$ siècle, Paris, Éditions de l'EHESS, 1997, p. 7-16, pour une approche méthodologique; Philippe Minard, « Faire l'histoire sociale des institutions: démarches et enjeux", Bulletin de la Société d'Histoire moderne et contemporaine, $\mathrm{n}^{\circ} 3-4,2000$, p. 119-123 pour une synthèse commode; Michel Cassan, «De l'État «moderne » à ses administrateurs «moyens", Histoire, Économie, Société, n 4, 2004, p. 467-472 pour une présentation de travaux récents sur «État et administrateurs de rang moyen à l'époque moderne ".

9. Christophe Blanquie, Les présidiaux de Daguesseau, Paris, Publisud, 2004, p. 59-107; Didier Catarina, Les justices ordinaires, inférieures et subalternes de Languedoc: essai de géographie judiciaire 1667-1789, Montpellier, Publications de l'Université de Montpellier III, 2003, p. 268-270 et 444-479 sur l'exploitation à l'échelle régionale des enquêtes de 1725-1728 et 1745.

10. Arch. dép. Puy-de-Dôme, 1 C 4856 : «État des officiers sujets à l'edit de mars 1709 » pour la généralité de Riom [25 juin 1709]; Arch. nat., $\mathrm{H}^{1} 158829^{\mathrm{c}}$, p. 7 : « Estat des recouvrements et des sommes payés par Messieurs les officiers compris aux rolles arestez au Conseil pour la compatibilité en consequence de l'Edit de mars 1709 » pour la généralité de Limoges du 12 mars 1712 ; Arch ; nat., K 891 (correspondance et tableaux sur les affaires extraordinaires, 1689-1712), $n^{\circ} 51$ (fo. 9-15) : état des offices de la généralité de la Rochelle du 30 avril 1712. Cette dernière nomenclature, conservée dans les dossiers de l'intendant des finances de Bercy, livre très probablement un nombre minimal et incomplet d'officiers cumulants, en ignorant ceux ayant déjà acquitté leur taxation car elle mentionne un rôle antérieur du 20 juillet 1709.

11. Arch. nat., K 891, $n^{\circ} 51$ (fol. 9-15) : le procureur du roi et substitut de Brouage Portier du Petitfief est aussi greffier et huissier de l'amirauté de Marennes tandis que Bégon cumule une charge de conseiller au parlement de Metz avec la fonction d'inspecteur générale de la marine ; Jean-Michel Deveau, « Aunis et Saintonge », Dictionnaire de l'Ancien Régime, Lucien Bély (sd.), Paris, PUF, 1996 (p. 112-114).

12. Georges Musset, Un parlement au petit pied. Le présidial de La Rochelle. Étude historique, La Rochelle, 1878, étudie surtout les conflits du siège avec la municipalité; Léopold-Gabriel Delayant, Du présidial de La Rochelle, La Rochelle, 1878, commente seulement l'ouvrage précédent. En revanche, les présidiaux de Riom et d'Aurillac bénéficient d'études utiles, Édouard Everat, $L a$ sénéchaussée d'Auvergne et siège présidial de Riom au XVIII siècle. Étude historique, Paris, Thorin, 1885 ; Jean Malmezat, Le Bailli des Montagnes d'Auvergne et le présidial d'Aurillac, Paris, 1941. Pour les présidiaux de Limoges et de Tulle, nous nous permettons de renvoyer à notre recherche, Vincent Meyzie, Les illusions perdues de la magistrature seconde. Les officiers « moyens » de justice en Limousin et en Périgord (vers 1665-vers 1810), Limoges, Pulim, 2006.

13. Michel Cassan, "Pour une enquête sur les officiers "moyens" de la France moderne", Annales du Midi, t. 108, janvier-mars 1996, p. 89-112, p. 92.

14. La prise en compte de la distinction cumul apparent-cumul véritable renforce la surreprésentation des officiers « moyens » de judicature parmi les cumulants : dans la généralité de Limoges, ils représentent 18 cas sur 33 officiers en situation véritable de cumul. Dans la généralité de Riom, l'exercice des recettes des tailles s'accomplit de manière majoritaire sous la forme de commissions.

15. BNF, Cinq cent Colbert 259 : évaluation des offices dans les élections de Limoges et de Tulle. 
16. Deux autres critères renforcent l'hypothèse d'une étanchéité professionnelle mais aussi sociale entre institutions judiciaires et financières dans la généralité de La Rochelle : l'absence d'officier du bureau des finances de La Rochelle parmi les cumulants, l'absence de patronymes communs entre membres des présidiaux et des élections.

17. David Parker, Class and State in Ancien Regime France. The road to Modernity?, London, Routledge, 1996, p. 173-182, souligne les insuffisances de la vision néo-webérienne de l'État des Lumières. Cette conception influence la synthèse de Carlo Capra, «Le fonctionnaire ", L'homme des Lumières, Michel Vovelle (sd.), Paris, Seuil, 1996 (p. 347-390).

18. Marie-Laure Legay, «Peut-on définir une élite administrative provinciale? Le cas artésien ( XVII'-XVIII siècles) », Revue du Nord, n 332, t. 81, octobre-décembre 1999, p. 705-721, p. 713-715 et 719-720 et Les États provinciaux dans la construction de l'État moderne aux XVII et XVIII ${ }^{e}$ siècles, Genève, Droz, 2001, p. 260-267, sur l'analyse comme administrateurs des députés généraux des États provinciaux du Nord de la France.

19. Philippe Minard, La fortune du colbertisme. État et industrie dans la France des Lumières, Paris, Fayard, 1998, p. 75-114.

20. Nicole Dyonet, «Les officiers de maréchaussée et les villes au XVIII'siècle », p.139-152 in M. Cassan, (sd.), op. cit., sur la réforme de 1720 ; Daniel Martin, « La maréchaussée au XVIII siècle. Les hommes et les institutions en Auvergne", Annales historiques de la Révolution française, janviermars 1980, p. 91-117 et «La soumission au «Centre », facteur d'identité ? », L'identité de l'Auvergne (Auvergne-Bourbonnais-Velay): mythe ou réalité historique. Essai sur une histoire de l'Auvergne des origines à nos jours, D. Martin (sd.), Nonette, Créer, 2002 (p.283-309, p. 296-298), sur la maréchaussée en Auvergne.

21. La ville des temps modernes de la Renaissance aux Révolutions, Emmanuel Le Roy Ladurie, (sd.), Paris, Seuil, 1998 (1 $1^{\text {ère }}$ éd. 1980), p. 166-167 ; W. Doyle, Venality. The Sale of Offices in Eighteenth Century France, Oxford, Clarendon Press, 1996, p. 26-57.

22. Arch. nat., $\mathrm{H}^{1} 158829^{\mathrm{C}}$, p. 4 : état de la maréchaussée du Limousin du 22 avril 1712 concernant le recouvrement des taxes selon l'édit de novembre 1701.

23. Luca Mannori, «Centralisation et fonction publique dans la pensée juridique de l'Ancien Régime : justice, police et administration ", L'administration de la France (1750-1940), Michel Pertué, (sd.), Orléans, Presses de l'Université d'Orléans, 1988 (p. 247-257).

24. Joël Hautebert, La justice pénale à Nantes au Grand Siècle. Jurisprudence de la sénéchaussée présidiale, Paris, Michel de Maule, 2001, p. 85-86.

25. Ce souci d'éviter une concentration abusive du pouvoir est attesté à la même époque chez l'intendant de la généralité de Bordeaux qui justifie le fait de ne pas choisir comme subdélégué le «lieutenant général et maire [de Périgueux]; c'aurait été donner trop d'autorité à la même personne [...] j'ai cru qu'il valait mieux tenir un contrepoids », Ferdinand Villepelet (éd.), « Le Périgord aux Archives nationales d'après les papiers du contrôle général des finances (1682-1727) », Bulletin de la Société Historique et Archéologique du Périgord, t. XXXV, 1908, p. 211-224, 259-274, 362-394, p. 264 : lettre du 28 mai 1701.

26. Arch. dép. Puy-de-Dôme, 1C 7066 : «Etat du Bailliage et Siege Presidial d'Aurillac du 13 aoust 1724 », «État des offices royaux existans dans l'election d'Aurillac au $1^{\mathrm{er}}$ avril 1725 ». L'office de lieutenant général a été acquis en 1644 par la famille Delort pour 45000 livres, l'office de président est vendu pour 15000 livres en 1717.

27. Claude Grimmer, Vivre à Aurillac au XVIII siècle, Aurillac, Gerbert, 1983, p. 70-80.

28. Arch. dép. Puy-de-Dôme, 1 C 7068 : état du présidial de Clermont [1723-1725]; Albert de Remagle, Dictionnaire généalogique. Familles d'Auvergne, Clermont, A.R.G.H.A, 1995-1996 (1 ère éd. 1941-1943), t. 2 ; Christophe Blanquie, op.cit., p.316-318, pour les évaluations des offices présidiaux d'Aurillac et de Clermont en 1728. 
29. Les cas de disjonction entre les offices possédés et les villes sont rares (exemples dans les tableaux I, II et III).

30. Bernard Lepetit, Les villes dans la France moderne (1740-1840), Paris, Albin Michel, 1988, p. 154-158.

31. Jean-Baptiste Bouillet (éd.), État de l'Auvergne en 1765 présenté à M. de l'Averdy par M. de Ballainvilliers dans Tablettes historiques de l'Auvergne, t. 7, 1846, p. 1-192; p. 100 : il note que « cette affluence de plaideurs [...] est la seule ressource de la ville ».

32. Robert Favier, op. cit., p. 67, à propos de la création en 1638 d'un présidial à Valence.

33. La différence entre les deux chiffres résulte des cas de réunion d'offices ou d'exercice par des commissions mais aussi de la prise en compte des offices vacants, peu nombreux dans la généralité d'Auvergne, Archives départementales Puy-de-Dôme, 1 C 7065 : lettre de l'intendant du 17 septembre 1724 au garde des Sceaux d'Armenonville.

34. Daniel Jousse, Traité de l'administration de la justice, Paris, Debure Père, 1771, t. 1, p. 142-164 et 512-515; Paul Louis-Lucas, Étude sur la vénalité des charges et fonctions, Ancien droit français-Droit intermédiaire, Paris, Challamel et Thorin, 1882, t. 2, p. 433-437 : «De l'incompatibilité ».

35. Jean-André Tournerie, Le présidial de Tours de 1740 à 1790, Études de la Faculté des Sciences juridiques et économiques de Tours, $\mathrm{n}^{\circ} 1,1975$, p. 24-29.

36. Lettre de l'intendant au contrôle général du 22 décembre 1699 citée par Christian Laroche, L'intendant Bernage en sa généralité de Limoges (1699-1702). Étude effectuée d'après la correspondance envoyée par l'intendant au Contrôle Général de 1699 à 1703, TER, Limoges, 1987, p. 140.

37. Lettre de l'intendant de Bérulle au contrôle général du 21 octobre 1685 sollicitant l'agrément pour de Fargues, « homme riche de 150000 livres, de probité, âgé de cinquante ans, et très estimé de la province » citée par Jean Malmezat, op. cit., p. 137-138.

38. "Mémoire sur la généralité de La Rochelle, de Michel Bégon », Georges Musset (éd.), Archives historiques de la Saintonge et de l'Aunis, 1875, t. II, p.17-174, p. 34-36; Mémoire sur l'état de la Généralité de Riom en 1697, dressé pour l'instruction du duc de Bourgogne par l'intendant Lefèvre d'Ormesson, Abel Poitrineau (éd.), Clermont-Ferrand, Institut d'Études du Massif Central, p. 173 ; «Mémoire sur la Généralité de Limoges dressé par Louis de Bernage, sgr. de Saint-Maurice, intendant (1698) », Alfred Leroux (éd.), Bulletin de la Société archéologique et historique du Limousin, t. XXXII, 1885, p. 149-258, p. 208-210 et 213.

39. Arch. nat., $\mathrm{H}^{1} 158844$ : le montant de la taxation s'élève à 450 livres pour une évaluation des finances entre 4000 et 10000 livres, à 900 livres entre 10000 et 15000 , à 1800 livres entre 15000 et 25000 , à 2700 livres entre 25000 et 40000 , à 3600 livres entre 40000 et 60000 et à 5400 livres au-delà de 60000 livres. Deux remarques sur cette distribution fiscale : fixée à une évaluation minimale de 4000 livres, elle délaisse la catégorie du cumul de petits offices; plafonnée à une taxation maximale, elle favorise relativement les détenteurs de charges coûteuses.

40. David D. Bien, «Les offices, les corps et le crédit d'État: l'utilisation des privilèges sous l'Ancien Régime ", Annales E.S.C., mars-avril 1988, n² 2, p. 379-404; Mark Potter, "Good Offices : Intermediation by Corporate Bodies in Early Modern French Public Finance ", The Journal of Economic History, 60-3, 2000, p. 599-626.

41. L'estimation de 140000 livres est obtenue par l'addition des sommes indiquées sur la liste de 1709 et une liste du 29 novembre 1712, Arch. dép. Puy-de-Dôme, 1 C 4862. Dans la généralité de La Rochelle, la taxation mise en 1712 sur 84600 livres.

42. Arch. dép. Puy-de-Dôme, 1 C 4863 : récépissé du 20 février 1717 pour Gabriel Servant, lieutenant général de police et procureur du roi au bailliage de Montferrand.

43. Arch. nat., $\mathrm{H}^{1} 158818^{\mathrm{A}}$, fo. $82 \mathrm{v}$. : rôle pour la confirmation de compatibilité du 14 juin 1712 pour la généralité de Caen ; Arch. nat., $\mathrm{H}^{1} 158818^{\mathrm{B}}$, p. 24 et 59 : état des affaires extraordinaires en 1711 et état du recouvrement pour la compatibilité du 26 août 1710 ; Arch. nat., $\mathrm{H}^{1} 158837^{\mathrm{B}}$, fo. $81 \mathrm{v}$. : état du recouvrement pour la compatibilité de mai 1712 pour la généralité de Moulins. 
44. Arch. dép. Puy-de-Dôme, 1 C 4862 : copie de la lettre de l'intendant des finances de Bercy à l'intendant d'Auvergne Turgot du 4 septembre 1713.

45. Correspondance des contrôleurs généraux des finances avec les intendants des provinces, Arthur de Boislisle (éd.), Paris, Imprimerie nationale, 1874-1897, t. II, p. 268: lettre de Montgeron, intendant de la généralité de Limoges du 7 février 1710; le mémoire de 1698 évoque les fondements familiaux de la fortune de Chirade, «fils d'un riche marchand et qui s'est marié richement ", Alfred Leroux (éd.), op. cit., p. 210.

46. Arch. dép. Haute-Vienne, $4 \mathrm{E}$ III 25 , notaire Debeaubreuil : inventaire après décès du 21 juillet 1710.

47. En 1704 et en 1709, les officiers de six élections de la généralité de Riom ont été obligés d'acquérir collectivement les offices d'élus contrôleurs anciens puis alternatifs et triennaux des quittances des receveurs des tailles pour les sommes globales de 23500 et 7250 livres, Arch. dép. Puy-de-Dôme, 1 C 4856 : rôle du 10 juin 1704 et rôle de modération du 26 mars 1709.

48. Christophe Blanquie, Justice et finance sous l'Ancien Régime. La vénalité présidiale, Paris, L'Harmattan, 2001, p. 173-175 et 181-185.

49. Arch. nat., $\mathrm{G}^{7} 346$ : « Mémoire des officiers qui exercent la police dans les principales villes de la généralité de Limoges » et lettre de l'intendant du 9 février $1690 ; \mathrm{AN}, \mathrm{H}^{1} 1588,29^{\mathrm{A}}$, p. 55 ; "Mémoire sur l'Angoumois par Jean Gervais, lieutenant criminel au présidial d'Angoulême», Gilles Babinet de Rencognes (éd.), [1864], p. 466 et 475.

50. Antoine Follain, «Les juridictions subalternes en Normandie. Entre service et commerce: honneur et perversité de la justice aux XVI ${ }^{e}$ et XVII ${ }^{e}$ siècles ", Annales de Normandie, 1999, n 5 , p. 539-566; Samuel Gibiat, «Les notaires royaux de Montluçon à l'époque moderne : l'institution, les offices, la pratique et les hommes", Revue historique, t. CCCIX/1, janvier 2004, p. 81-120, p. 99-101, note un nombre significatif de notaires cumulants.

51. Marc Perrichet, «Les receveurs des tailles de la généralité de Caen, 1661-1715 ", Les finances en province sous l'Ancien Régime, Journée d'étude de Bercy 1998, Françoise Bayard (sd.), Paris, CHEFF, 2000 (p. 29-122, p. 56 pour la citation). À Tulle, le président du présidial et receveur des tailles Jean-Baptiste Jaucen incarne les avantages de ce cumul, facteur de considération sociale et d'enrichissement économique pour l'officier et sa famille, Vincent Meyzie, op. cit., p. 257-262.

52. Arch. dép. Puy-de-Dôme, 1 C 7068 : états des offices dans l'élection de Clermont [1723-1725]. En 1717, Chamaret vend l'office de greffier à ce prix et dans la décennie 1710 deux charges d'élus sont vendues pour ce montant.

53. Arch. dép. Puy-de-Dôme, 1 C 4862: rôle du 29 novembre 1712 indiquant une liste complémentaire de 23 officiers royaux cumulants; Arch. dép. Puy-de-Dôme, 2 E 01585 : livre de comptes de Courtin [1711-1731] avec un «Estat des sommes pour lesquelles nous sommes employes mon pere et moy dans l'estat des finances de l'année 1708 " et les "gages, augmentations et appointements en qualité de commissaire de la marine » pour 1711 ; Édouard Everat, Le Bureau des finances de Riom (1551-1790), Riom, Jouvet, 1900, p. 95-106, 278-286 et 396-400 («notes généalogiques»). Édouard Everat note que «les Courtin avaient été en Auvergne les agents du ministère de la marine », ibid, p. 105-106, notamment pour l'approvisionnement en bois de construction.

54. Édouard Everat, ibid, p. 374-379.

55. Ibid, p. 188-189: remontrance au roi du bureau des finances de Riom du 16 juin 1787 indiquant l'anoblissement réussi de six familles depuis sa création.

56. L'attractivité supérieure des offices de finance en général et des offices anoblissants en particulier sur les charges de justice constitue un thème récurrent dès la première moitié du XVIII ${ }^{e}$ siècle, Arch. dép. Puy-de-Dôme, 1 C 7068 : état du présidial de Clermont [1723-1725]; Louis Vialatte, Rossignol, intendant de la généralité de Riom et province d'Auvergne (1734-1750), Aurillac, 1924, p. 19-35. 
57. Philippe Rosset, Les officiers du Bureau des Finances de Lille (1691-1700), Paris-Genève, Droz, 1991, p. 122-135, sous-estime les motivations financières des acquéreurs.

58. Arch. dép. Puy-de-Dôme, 1C 7071 : «État des offices royaux qui existent actuellement dans le département de M. Carraud, subdélégué à Riom » [1723-1725].

\section{AUTEUR}

\section{VINCENT MEYZIE}

Université de Limoges/CERHILIMM 